REVISTAS DE LA FAHCE

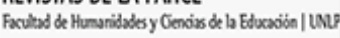

की
Educación Física y Ciencia

ISSN: 1514-0105

ISSN: 2314-2561

revistaefyc@fahce.unlp.edu.ar

Universidad Nacional de La Plata

Argentina

\title{
Pilates: efeitos na forca e imagem corporal de mulheres sedentárias
}

Souza, Catiane; Detogni Schmit, Emanuelle Francine; Wagner Neto, Edgar Santiago; Couto de Sá, Cloud Kennedy; Loss, Jefferson Fagundes

Pilates: efeitos na forca e imagem corporal de mulheres sedentárias

Educación Física y Ciencia, vol. 22, núm. 1, 2020

Universidad Nacional de La Plata, Argentina

Disponible en: http://www.redalyc.org/articulo.oa?id=439963095010

DOI: https://doi.org/10.24215/23142561e118

Esta obra está bajo una Licencia Creative Commons Atribución-NoComercial-Compartirlgual 4.0 Internacional. 
Artículos

\section{Pilates: efeitos na forca e imagem corporal de mulheres sedentárias}

Pilates: effects on strength and body image of sedentary women

Catiane Souza

Centro Universitário Maurício de Nassau, Brasil

catiane.souza@ufrgs.br

DOI: https://doi.org/10.24215/23142561e118

Redalyc: http://www.redalyc.org/articulo.oa?

Emanuelle Francine Detogni Schmit

Cesuca-Complexo de Ensino Superior de Cachoeirinha,

Brasil

Edgar Santiago Wagner Neto

Universidade Federal do Rio Grande do Sul, Brasil

Cloud Kennedy Couto de Sá

Universidade Estadual de Feira de Santana, Brasil

Jefferson Fagundes Loss

Universidade Federal do Rio Grande do Sul, Botsuana

Recepción: 24 Septiembre 2018

Aprobación: 20 Diciembre 2019

\section{Resumo:}

O presente estudo teve por objetivo avaliar a imagem corporal e a força de praticantes inexperientes ao longo de 30 sessões de Pilates. Participaram 19 mulheres sedentárias sem contato prévio com o método e idade entre 21 e 35 anos, submetidas a 30 sessões de Pilates em turmas de quatro alunas, duas vezes por semana. As avaliações foram realizadas em cinco momentos: antes do período controle de um mês, antes da intervenção, após dez sessões, após 20 sessões e após 30 sessões. Foi avaliada a força de preensão palmar como representativa da força geral, de forma complementar a força dos extensores do tronco e a imagem corporal que foi avaliada pela diferença da imagem de corpo considerada atual e ideal, a partir de uma Escala de Imagem Corporal. Ao longo de trinta sessões de método Pilates a força dos extensores da coluna apresentou diferença estatística e tamanho de efeito grande entre o período pré e pós-intervenção $(. T=9,50 ; . r=-0,537 ; \mathrm{z}=-3,311 ; \mathrm{p}=0,001)$. A imagem corporal e a preensão palmar não foram alteradas.

Palavras-CHaVE: Pilates, Saúde mental, Saúde física, Imagem corporal, Força lombar.

\section{Abstract:}

The present study had the objective to assess the body image and overall strength of inexperienced practitioners along 30 Pilates sessions. 19 sedentary woman, naive to the method, with ages between 21 and 35 were submitted to 30 classic Pilates classes, in groups of four, twice a week. The evaluations took place in five occasions: before a month-long control period, before the intervention and after 10,20 and 30 sessions. Gripstrenght was evaluated as representative for overall strength, complimentary to trunk extensors and body image assessed, with a body image scale, by the difference between the current self-assigned body image and the one considered ideal. Over 30 classical Pilates sessions trunk extensor strength presented statistical difference and big effect size between pre and post-intervention periods $(T=9,50 ; r=-0,537 ; z=-3,311 ; p=0,001)$. Body image and gripstrenght did not differ.

KEYwORDS: Pilates, Mental health, Physical health, Body image, Lumbar strength.

\section{INTRODUÇÃO}

A disseminação do método Pilates, a nível mundial é a cada dia mais reconhecida (Latey, 2001; Ferreira et al., 2007; Di Lorenzo, 2011; Costa, Roth, \& Noronha, 2012). Seu sistematizador, Joseph Humbertus Pilates, 
afirmava que não basta dizer que a saúde é uma condição normal, também é um dever alcançá-la e mantêla, propondo-se assim a trabalhar o corpo como um todo, proporcionando saúde no seu sentido mais amplo: físico e mental (Pilates, \& Miller, 1934).

A saúde mental, segundo a World Health Organization (WHO, 2005), pode ser entendida como um estado de ser no qual o indivíduo realiza as suas próprias capacidades, pode fazer face ao estresse normal da vida, trabalhar de forma produtiva e frutífera, e é capaz de dar um contributo para a sua comunidade. Uma das manifestações mais comuns de problemas relacionados à saúde mental na sociedade consiste na alteração da autoimagem (Godoy, 2002). A autoimagem pode ser entendida como sinônimo de imagem corporal, a qual corresponde à figuração de nosso corpo formada em nossa mente acerca das dimensóes corporais, bem como às informações do nível de satisfação ou rejeição corporal, ou seja, ao modo pelo qual o corpo apresenta-se para nós, inclusive como uma representação de desejos, emoções e socialização com os outros indivíduos (Schilder, 1999; Quadros et al., 2010). Uma imagem corporal negativa, isto é, uma insatisfação com o corpo, pode levar a diversos comportamentos impróprios, além de depressão, desordens alimentares e atividades físicas em excesso (Oliveira, Bosi, Vigário, \& Vieira, 2003). A insatisfação com a imagem corporal em mulheres é maior do que em homens (Damasceno, Lima, Vianna, Vianna, \& Novaes, 2005), podendo ser considerada um problema de grande escala (Sante, \& Pasian, 2011).

Em relação à influência do método Pilates na imagem corporal, a literatura é contraditória. Cruz-Ferreira et al. (2011) encontraram melhora significativa na percepção da aparência física, bem como em outras percepções sobre o corpo e sobre como ele é visto por outras pessoas, enquanto Serafini, Vargas, Junior e Tocha (2014) concluíram que o Pilates não é eficaz para reduzir a insatisfação com a imagem corporal. Ainda, foi encontrado um estudo considerado inconclusivo pelos autores, sobre como o indivíduo se percebe ao longo do treinamento (Neumark-Sztainer, Eisenberg, Wall, \& Loth, 2011).

No que se refere ao aspecto físico, uma aptidão que permanece inconclusiva com relação aos efeitos do Método Pilates é a força. Força muscular, segundo o American College of Sports Medicine (ACSM, 2011). também pode ser denominada vigor muscular ou potência muscular e está relacionada com a capacidade de realizar atividades que exijam altos níveis de força muscular. Os efeitos das sessões de Pilates sobre a força muscular também apresentam resultados contraditórios Kolyniak, Cavalcanti e Aoki (2004) apontaram resultados positivos, enquanto Christopher, Casebolt, Silver e Kwon (2007) não obtiveram diferença. Ainda, embora sem o objetivo específico de investigar a força muscular, são encontrados estudos que abordam esta aptidão física indiretamente, como por exemplo, avaliando a força por meio da pressão abdominal (DonahoeFilmore et al., 2007) o que não condiz com medidas válidas para esse fim (ACSM, 2011).

Pode-se ainda reconhecer a inferência da saúde física e da saúde mental no método Pilates em uma frase amplamente difundida, atribuída por diversos autores a Joseph Pilates: Em dez sessões você sentirá a diferença, em 20, você verá a diferença e em 30, você terá um novo corpo (Dufton, 2003; Siler, 2008). Como forma de dar suporte científico a esta afirmação empírica, poderíamos afirmar que "sentir", "ver" e "ter" um corpo referem-se especificamente a variáveis de autoimagem e aspectos físicos, como a força. Sendo assim o objetivo deste estudo é avaliar a imagem corporal e a força de praticantes inexperientes ao longo de 30 sessões de Pilates.

\section{METODOLOGIA}

Foi conduzido um estudo quase experimental, sem um grupo controle, mas com um período controle de quatro semanas. 


\section{Amostra}

Foram selecionadas 21 mulheres de acordo com os seguintes critérios de elegibilidade: ter entre 21 e 35 anos; ser sedentária, ou seja, há mais de 6 meses não seguir as recomendações da OMS (de 150 minutos semanais de atividade física moderada); não ter contato prévio com o método; não ter contra-indicação médica à pratica de atividade física; não ter tido parto ou gestação os últimos seis meses. Foram critérios de exclusão: faltar a duas sessões seguidas ou a quatro sessões alternadamente sem as recuperarem na mesma semana e não comparecer a todas as sessões avaliativas. Foi solicitado que não houvesse alterações na alimentação, nem mesmo alterações no nível de atividade cotidianas, como por exemplo, caminhadas para pegar o ônibus, ou tarefas de limpeza.

O projeto foi aprovado no Conselho de Ética em Pesquisa da Universidade onde foi realizado, seguiu as disposições da resolução 466/12 do Conselho Nacional de Saúde e foi conduzido de acordo com a Declaração de Helsinque.

\section{Intervenção}

As 30 sessões foram realizadas no Laboratório de Pesquisa e Exercício da Universidade Federal do Rio Grande do Sul, ministradas por uma bacharel em educação física com formação no método Pilates, em grupos de, no máximo, quatro pessoas, duas vezes por semana com duração média de 50 minutos, seguindo um protocolo de mat baseado na sequencia clássica e nos exercícios proposto por Siler (2008). O protocolo foi desenvolvido pela instrutora que aplicaria as sessões juntamente com uma experiente professora de formações de Pilates clássico, teacher trainer da Power Pilates, PMA certified teacher, cuja escola possui Registry School. O protocolo prevê a ordem de realização dos exercícios, com progressões a cada 5 aulas, de forma que todos os grupos tivessem incrementos de dificuldade de forma homogênea (Tabela 1). Para contornar as limitações de cada indivíduo, sem deixar de seguir o protocolo, exercícios preparatórios, adaptados e/ou desmembrados foram incluídos em algumas sessões. 
TABELA 1

Protocolo para 30 sessões de Mat Pilates

\begin{tabular}{|c|c|c|}
\hline Exercício & Repetições & Sessões \\
\hline the hundred & 100 & 1 a 30 \\
\hline roll up & 3 a 5 & 1 a 30 \\
\hline single leg circles & 3 a 5 & 1 a 30 \\
\hline rolling like a ball & 5 a 6 & 1 a 30 \\
\hline single leg stretch & 3 a 5 & 1 a 30 \\
\hline double leg stretch & 5 & 1 a 30 \\
\hline single straight leg stretch & 3 & 11 a 30 \\
\hline double straight leg stretch & 3 & 11 a 30 \\
\hline crisscross & 5 & 16 a 30 \\
\hline spine stretch forward & 3 & 1 a 30 \\
\hline open leg rocker & 5 & 16 a 30 \\
\hline corkscrew & 3 & 16 a 30 \\
\hline saw & 3 & 11 a 30 \\
\hline single leg kicks & 3 & 1 a 30 \\
\hline doble leg kicks & 6 & 21 a 30 \\
\hline neck pull & 3 & 16 a 30 \\
\hline swan & 3 a 5 & 1 a 30 \\
\hline side kicks/front back & 3 & 11 a 30 \\
\hline side kicks/up down & 3 a 5 & 6 a 30 \\
\hline side kicks/small circles & 3 a 5 & 6 a 30 \\
\hline side kicks/inner-thigh lifts & 3 a 5 & 16 a 30 \\
\hline side kicks/heel beats & 3 a 5 & 21 a 30 \\
\hline teaser & 3 a 5 & 6 a 30 \\
\hline swimming & 10 & 1 a 30 \\
\hline pull up & 3 & 1 a 30 \\
\hline the seal & 3 a 5 & 21 a 30 \\
\hline zip up & 3 a 5 & 1 a 30 \\
\hline chest expansion & 4 & 1 a 30 \\
\hline shaving the head & 5 & 1 a 30 \\
\hline amm circles & 3 a 5 & 1 a 30 \\
\hline biceps curl I & 3 a 5 & 1 a 30 \\
\hline biceps curl II & 3 a 5 & 1 a 30 \\
\hline tríceps extension & 3 a 5 & 21 a 30 \\
\hline the bug & 3 a 5 & 21 a 30 \\
\hline circles on the wall & 5 & 1 a 30 \\
\hline sliding down the wall & 3 & 1 a 30 \\
\hline rolling down the wall & 5 & 1 a 30 \\
\hline saida the wall & & 1 a 30 \\
\hline
\end{tabular}

\section{Instrumentos}

O peso e a estatura foram avaliados através de uma balança digital portátil com sensibilidade de $100 \mathrm{~g}$ (TechLine, São Paulo, São Paulo) e de uma fita métrica com sensibilidade de $1 \mathrm{~mm}$ (Sanny, São Bernardo do 
Campo, São Paulo). Para a avaliação da imagem corporal foi utilizada a Escala de Imagens Corporais (Souza et al., 2017), desenvolvido especificamente para mulheres brasileiras, um instrumento de baixo custo e rápida aplicação/avaliação. Para avaliar força, foram utilizados um dinamômetro palmar e um dinamômetro lombar.

\section{Procedimentos}

Todas as avaliações foram realizadas pela mesma equipe, com metodologia cega, sem que o professor que ministrou as aulas participasse ou tivesse acesso aos dados das avaliações, e sem que a equipe avaliadora tivesse contato com as participantes no período das aulas.

No primeiro dia de coleta, todas as participantes assinaram o Termo de Consentimento Livre e Esclarecido. Então, foram mensurados o peso e estatura, e em seguida foram avaliadas a imagem corporal e a força, assim como em todos os demais momentos de avaliação: após o período controle, após dez, 20 e 30 sessões.

$\mathrm{Na}$ avaliação da imagem corporal, o instrumento é composto não só pelas silhuetas, mas também por duas questóes objetivas: "Qual imagem representa o seu corpo atual?" e "Qual imagem representa o corpo que você gostaria de ter?". A diferença entre a resposta da segunda e a resposta da primeira questão expressa o nível de insatisfação corporal. A insatisfação pode ser assim expressa numericamente, partindo de zero, mesma resposta em ambas questões, ou seja, nenhuma insatisfação, chegando a dez, máxima insatisfação. O resultado numérico pode ainda ser positivo ou negativo, representando o desejo de ser maior ou o desejo de ser menor, respectivamente. No presente estudo foram extraídas duas informações: (1) figuração corporal, dada pela resposta da primeira questão que aborda a silhueta auto-referida como representativa do corpo atual e (2) a satisfação com a imagem corporal, atribuída à diferença entre o corpo apontado como o atual e o corpo autoreferido como ideal (Souza et al., 2017).

Para variável força, foi assumido o teste de preensão palmar da mão dominante como representativo da força geral dos indivíduos (Madrid et al., 2010). O teste foi realizado com o indivíduo sentado, com cotovelo fletido a $90^{\circ}$, sem que houvesse contato do dinamômetro com outro segmento corporal. Para uma informação adicional a cerca da força, foi utilizado também um dinamômetro lombar. Os indivíduos foram instruídos a ficar em pé sobre a plataforma do dinamômetro, com os joelhos estendidos, tronco flexionado à frente, nesta posição o cabo do aparelho foi ajustado de acordo com a altura do indivíduo, ficando próximo ao joelho. O sujeito deveria segurar a manopla com os braços estendidos e aplicar o máximo de força possível no movimento de extensão da coluna. Em ambos os testes foi adotado o maior valor em três testes realizados, tendo, no mínimo dois minutos de intervalo entre as tentativas. Todas as tentativas contaram com incentivo verbal para melhor realização de força.

\section{ANÁLISE ESTATÍSTICA}

A análise estatística foi realizada no software SPSS versão 20.0 for Windows. Foi utilizada estatística descritiva, média, desvio padrão e frequência em percentual. Foi verificada a normalidade dos dados pelo teste de Shapiro-Wilk e a esfericidade pelo teste de Mauchly. Após confirmação dos pressupostos, nos dados de força, foram aplicadas ANOVAs para medidas repetidas, com um único fator, sendo este o tempo, compreendendo cinco momentos de avaliação (período controle, pré-treinamento, após 10 sessóes, após 20 sessões e após 30 sessóes). Para os dados da escala de imagem corporal, foi utilizada a ANOVA de Friedman, levando em consideração os mesmos cinco momentos.

Quando apontada diferença significativa pela ANOVA, foram realizados testes post hoc de comparação aos pares pelo teste de Wilcoxon, com correção de Bonferroni. Para as combinações que apresentaram diferença, foi calculado o tamanho de efeito através do coeficiente $r$ da correlação de Pearson, o qual foi 
interpretado conforme proposto em Field (2009): $r=0,10$ um efeito pequeno (explica 1\% da variância total), $r=0,30$ um efeito médio (explica 9\% da variância total) e $r=0,50$ um efeito grande (explica $25 \%$ da variância total). O nível de significância adotado foi $\mathrm{p}<0,05$.

\section{RESULTADOS E DISCUSSÃO}

Após uma perda amostral de dois indivíduos, que excederam o número de faltas previsto, o estudo foi concluído com 19 mulheres (idade $26,8 \pm 3,5$ anos, peso $57,9 \pm 6,8 \mathrm{~kg}$ e estatura $160 \pm 7 \mathrm{~cm}$ ).

A imagem corporal não mudou ao longo das sessões de Pilates, $\chi 2(2)=1,58 ; p=0,824$. A silhueta apontada como atual ficou entre as opções dois a oito em todas as avaliações, enquanto a silhueta apontada como ideal teve uma variação menor, ficando entre as opções três a seis.

A satisfação corporal, não variou no período controle e ao longo das 30 sessões de Pilates $\chi 2(2)=8,86$; $\mathrm{p}=0,580$ (Tabela 2$)$.

TABELA 2

Percentual dos escores de satisfação corporal em cada uma das avaliações

\begin{tabular}{|c|c|c|c|c|c|c|}
\hline Satisfação & $\begin{array}{c}\text { pré } \\
\text { controle } \\
(\%)\end{array}$ & $\begin{array}{c}\text { pré } \\
\text { intervenção } \\
(\%)\end{array}$ & $\begin{array}{c}10 \text { sessões } \\
(\%)\end{array}$ & $\begin{array}{c}20 \text { sessões } \\
(\%)\end{array}$ & $\begin{array}{c}30 \text { sessões } \\
(\%)\end{array}$ & $\begin{array}{c}\text { TOTAL } \\
(\%)\end{array}$ \\
\hline-3 & 33 & 24 & 18 & - & 6 & 15 \\
\hline-2 & 8 & 35 & 35 & 41 & 35 & 33 \\
\hline-1 & 17 & - & 12 & 24 & 18 & 14 \\
\hline 0 & 25 & 18 & 18 & 18 & 29 & 21 \\
\hline 1 & 8 & 6 & 18 & 18 & - & 10 \\
\hline 2 & 8 & 12 & - & - & 6 & 5 \\
\hline 4 & - & - & - & - & 6 & 1 \\
\hline
\end{tabular}

O percentual de participantes satisfeitas com o corpo atual se manteve em torno de $20 \%$ ao longo de todos os momentos de avaliação, enquanto o predomínio entre as participantes insatisfeitas sempre foi na direção de diminuir a silhueta (em torno de 60\%). Estes resultados estão de acordo com a literatura, visto que a maior parte das mulheres tem o desejo de serem mais magras (Novaes y Vilhena, 2003), e em menor escala observase também um desejo por corpos maiores do que elas enxergam ter no momento da avaliação (Bosi, Luiz, Morgado, Costa, \& Carvalho, 2006).

Quando a figuração corporal foi comparada ao longo dos cinco momentos avaliados, não foi encontrada diferença estatística, sugerindo que 30 sessões de Pilates não são suficientes para alterar a imagem corporal das participantes. Estes resultados concordam com os achados de Serafini et al. (2014), que utilizaram um instrumento semelhante para avaliar 51 mulheres sedentárias após nove sessões de Pilates. No que se refere ao nível de satisfação corporal, ao longo das avaliações, $22 \%$ das mulheres apresentaram a mesma imagem como real e ideal (Tabela 2). No estudo de Miranda, Filgueiras, Mockdece, Teixeira e Ferreira (2012), 24,4\% dos sujeitos apresentou satisfação com a imagem corporal, porém mais de $45 \%$ da amostra era composta também por homens, e a literatura aponta que mulheres são mais insatisfeitas que homens (Damasceno, Lima, Vianna, Vianna, \& Novaes,2005).

As silhuetas intermediárias (cinco, seis e sete) foram indicadas em um maior número de respostas, totalizando mais de $50 \%$ em todas as avaliações. A silhueta apontada como ideal ficou distribuída entre as silhuetas três e seis, sendo a três com maior ocorrência em todas as avaliações, tendo $18 \%$ de frequência no 
total das avaliações, como real e $48 \%$ de referência como corpo ideal. Dentre as figuras apontadas em cada uma das questões, a figura quatro foi a que teve menor incidência nas respostas, o que pode ser explicado por essa figura representar um padrão corporal razoavelmente incomum nas mulheres brasileiras, fugindo ao padrão do "corpo violão" (Maldonado, 2006), ainda que na avaliação pré-controle, essa figura tenha apresentado o mesmo percentual de incidência que as figuras dois, cinco e oito, e na pré-intervenção, a mesma quantidade de ocorrências que a dois e a sete. Indicando que, mesmo mulheres que não se vêem com as proporções corporais idealizadas no Brasil desejam um corpo com tais características.

Os resultados referentes à força indicam que trinta sessões de mat Pilates aparentemente não alteram a força geral das praticantes, avaliada pelo teste de preensão palmar, que não apresentou diferença entre os cinco momentos avaliados $\mathrm{F}(1,78 ; 31,95)=2,587 ; \mathrm{p}=0,584$ (Figura 1). Sabe-se que a força de preensão palmar é correlacionada com a circunferência do antebraço (Madrid et al., 2010), a qual não modificou antes e depois da intervenção, indicando que os resultados encontrados devem ser interpretados com cautela, principalmente ao se considerar o aumento encontrado na força da musculatura lombar que mudou significativamente ao longo das 30 sessões de intervenção $\chi 2(4)=20,30 ; p=0,001$. O teste do dinamômetro lombar das participantes não sofreu alterações significativas do período controle até o início da pré-intervenção $T=51,50, r=-0,240(\mathrm{z}=-1,481 ; \mathrm{p}=0,072)$, porém foi encontrada diferença significativa comparando os resultados pré-intervenção e 20 sessões $T=9 ; r=-0,562(\mathrm{z}=-3,464 ; \mathrm{p}=0,001)$, bem como na comparação entre pré-intervenção e 30 sessões $(T=9,50 ; r=0,537(\mathrm{z}=-3,311 ; \mathrm{p}=0,001)$ (Figura 2).

\section{FIGURA 1}

Força geral de praticantes de Pilates, avaliada pela preensão palmar.

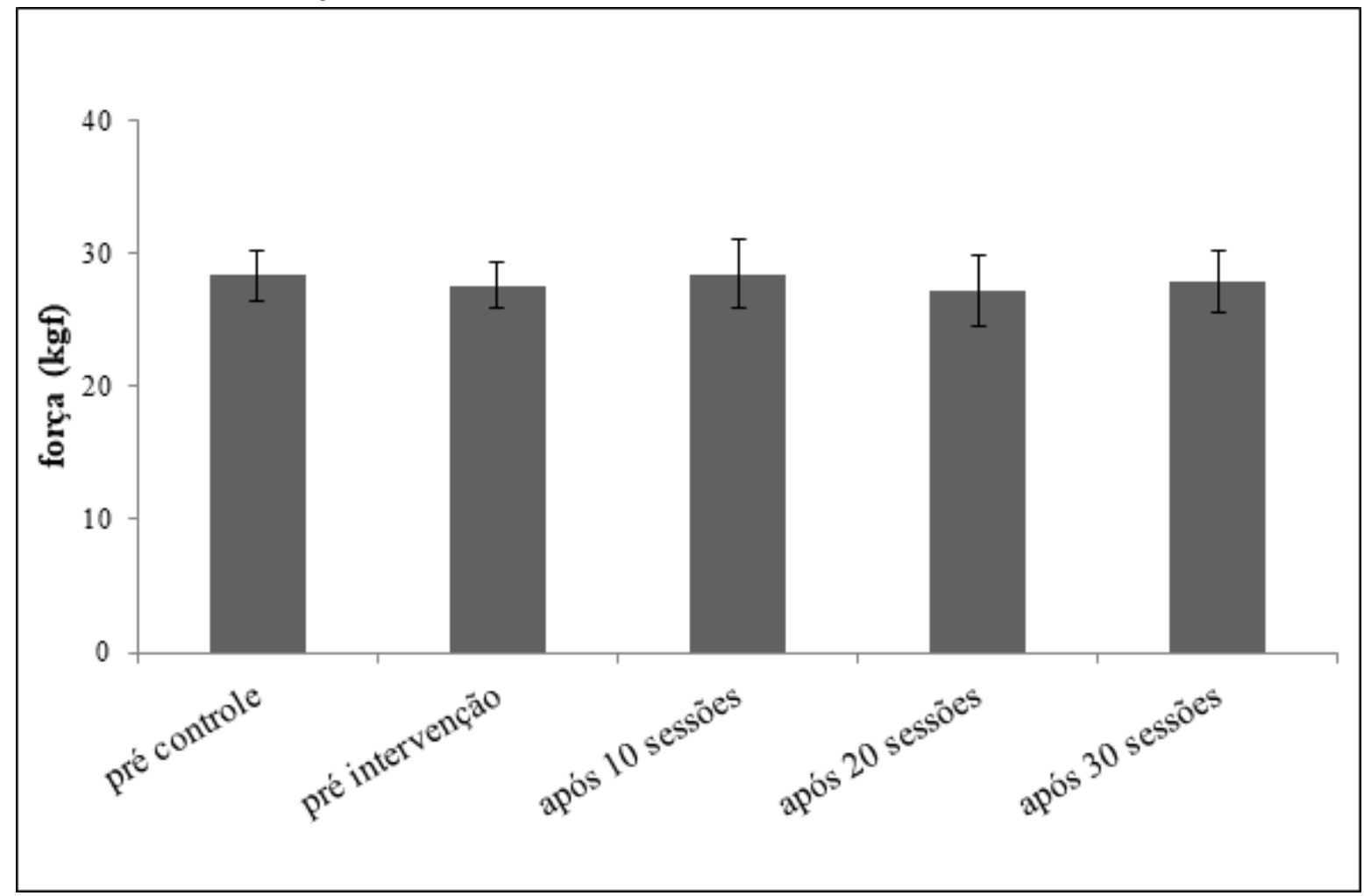

Fonte: a autora. 
FIGURA 2

Força dos extensores do tronco de praticantes de Pilates, avaliada por dinamômetro lombar.

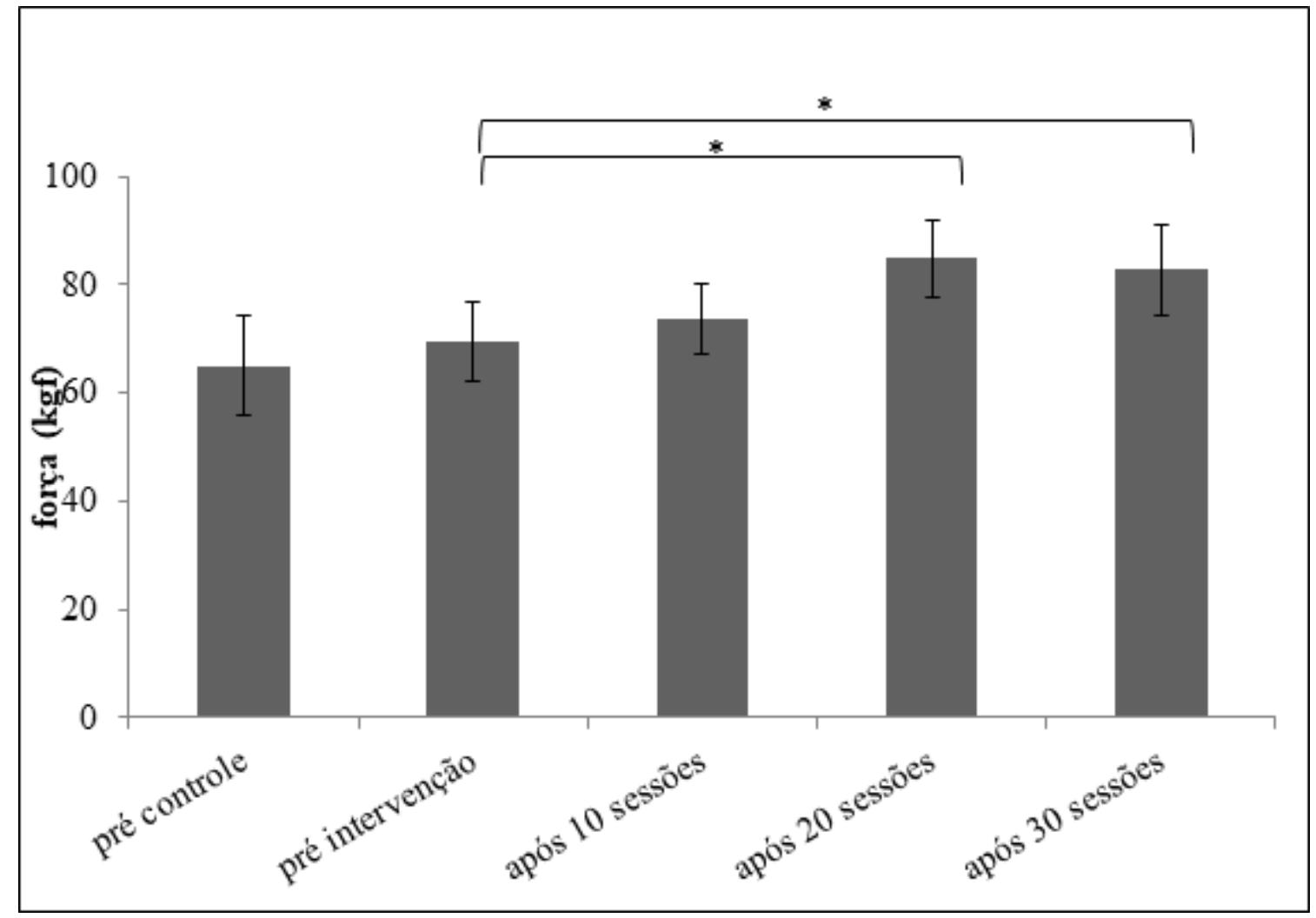

Fonte: a autora

A maior parte dos exercícios do método demanda mais os flexores do que os extensores do tronco, de forma que, essa musculatura foi também menos estimulada ao longo da intervenção do presente estudo, se comparada a outros grupos musculares. Os resultados encontrados na força lombar podem ser explicados, não só pelos exercícios do protocolo que tem essa musculatura como agonista (swan, swimming e pull up), mas também pelos estímulos constantes durante as sessões, ao princípio da centralização (Rossi et al., 2014): a musculatura extensora do tronco, juntamente com os abdominais, assoalho pélvico, flexores e extensores do quadril, forma o power house (Muscolino \& Cipriani, 2004), região considerada o centro físico de onde emanam todos os movimentos corporais (Gallagher y Kryzanowska, 2000). Especula-se ainda que, em grupos musculares mais demandados nas aulas, como os flexores do tronco, possa ser encontrado incremento de força ainda maior do que nos extensores do tronco.

Christopher, Casebolt, Silver e Kwon (2007) não encontraram diferença significativa entre o grupo intervenção e o grupo controle, no torque máximo isométrico dos abdutores do quadril, dos flexores e dos extensores do tronco. Já os achados de Kolyniak, Cavalcanti e Aoki (2004) corroboram os resultados referentes à força lombar do presente estudo, afirmando que o método mostrou-se uma eficiente ferramenta para o fortalecimento da musculatura extensora do tronco, atenuando o desequilíbrio entre a função dos músculos envolvidos na extensão e flexão do tronco.

Esses resultados conflitantes na literatura podem ser explicados por possíveis diferenças no protocolo de exercícios utilizado, fato esse que pode ter refletido um problema comum na prática do método: a escolha dos exercícios e de suas possíveis variações muitas vezes é baseada em orientações transmitidas informalmente dentre os instrutores (Souza, Cantergi, Mendonça, Kennedy, \& Loss, 2012). Sugerem-se novos estudos com diferentes frequências e maior tempo de duração para validar os achados descritos, bem como a avaliação desses parâmetros em diferentes populações. Sugere-se ainda que a preensão palmar seja 
avaliada em praticantes que realizem suas aulas em equipamentos, onde há exigência para preensão em diversos exercícios, de forma a esclarecer melhor a relação dessa variável com o treinamento de Pilates.

\section{CONCLUSÕES}

Ao longo de trinta sessões de método Pilates realizado em pequenos grupos 2 vezes por semana, houve efeito apenas na força de extensores do tronco, demonstrando que a partir de 20 sessões realizadas na frequência semanal adotada, já se pode encontrar alterações nessa variável. A imagem corporal e a preensão palmar não foram alteradas. Esses achados são de extrema relevância clínica, uma vez que oferecem um direcionamento concreto aos instrutores quanto à expectativa do incremento de força nos extensores lombares em mulheres sedentárias. Além disso, auxiliam no melhor entendimento científico acerca do método Pilates.

\section{REFERÊNCIAS}

American College of Sports Medicine (ACSM) (2011). Manual do ACSM para avaliação da aptidão física relacionada à saúde. Rio de Janeiro: Guanabara Koogan.

Bosi, M. L. M, Luiz, R. R, Morgado, C. M. C, Costa, M. L. S \& Carvalho, R. J. (2006). Auto percepção da imagem corporal entre estudantes de nutrição: um estudo no município do Rio de Janeiro. Jornal Brasileiro de Psiquiatria 55(2). Disponível em: http://www.scielo.br/scielo.php?pid=S0047-20852006000200003\&script=sci_abstrac t\&tlng=pt

Christopher, G., Casebolt, J., Silver, T. \& Kwon, Y. (2007) Effects of a pilates exercise program on core strenght in females. ISBS-Conference Proceedings Archive. Disponível em: https://www.ncbi.nlm.nih.gov/pmc/articles/P MC5005852/

Costa, L. M. R., Roth, A. \& Noronha, M. (2012) O método pilates no Brasil: uma revisão de literatura. Arquivos Catarinenses de Medicina 41(4), p. 87-92. Disponível em: http://www.acm.org.br/revista/pdf/artigos/1151.pdf

Cruz-Ferreira, A., Fernandes, J., Gomes, D., Bernardo, L. M., Kirkcaldy, B. D., Barbosa, T. M. \& Silva, A. (2011) Effects of Pilates-based exercise on life satisfaction, physical self-concept and health status in adult women. Women Health, 51(3) 240-55. Disponível em: https://pdfs.semanticscholar.org/5a78/99cad99ea4eecd163019ae50f42 d02c4b2a2.pdf

Damasceno, V. O, Lima, J. R. P, Vianna, J. M, Vianna, V. R. A \& Novaes, J. S. (2005) Tipo físico ideal e satisfação com a imagem corporal de praticantes de caminhada. Revista Brasileira de Medicina do Esporte. 11(3). Disponível em: http://www.scielo.br/scielo.php?script=sci_arttext\&pid=S1517-86922005000300006

Di Lorenzo, C. E. (2011) Pilates: What Is It? Should It Be Used in Rehabilitation? Sports Health, 3(4) 352. Disponível em: https://www.ncbi.nlm.nih.gov/pubmed/23016028

Donahoe-Fillmore B, Hanahan N, Mescher M, Clapp D, Addison N \& Weston C. (2007). The effects of a home Pilates program on muscle performance and posture in healthy females: a pilot study.Journal of Women's Health Physical Therapy. 31 6-11. Disponível em: http://ecommons.udayton.edu/cgi/viewcontent.cgi?article=1026\& context $=$ dpt_fac_pub

Dufton (2003), J. The Pilates Difference. Basic Health Pubn Incorporated. London, Great Britain.

Ferreira, C. B., Aidar, F. J., Novaes, G. D. S., Vianna, J. M., Carneiro, A. L \& Menezes, L. D. S. (2007). O método Pilates sobre a resistência muscular localizada em mulheres adultas. Motricidade, 3(4) 76-81. Disponível em: h ttp://www.redalyc.org/pdf/2730/273020485007.pdf

Field, A. (2009) Descobrindo a estatística usando o SPSS-2. Bookman. Porto Alegre, Brasil.

Gallagher, S. y Kryzanowska, R. (2000) O Método Pilates de Condicionamento Físico. 3 ed. The Pilates ${ }^{\circ}$ Studios Brasil.

Godoy, R. F.(2002) Benefícios do exercício físico sobre a área emocional. Movimento (ESEF/UFRGS) 8(2), 7-15. Disponível em: http://www.seer.ufrgs.br/Movimento/article/download/2639/1265 
Kolyniak, I. E. G., Cavalcanti, S. M. B. \& Aoki, M. S. (2004) Isokinetic evaluation of the musculature involved in trunk flexion and extension: Pilates@ method effect. Revista Brasileira de Medicina do Esporte, 10(6) 487-493. Disponível em: http://www.scielo.br/scielo.php?pid=S1517-86922004000600005\&script=sci_arttext\&tlng= en

Latey, P. (2001) The Pilates method: History and philosophy. Journal of Bodywork and Movement Therapies, 5(4), 275-282. Disponível em: http://activepilates.com.br/producoes/LATEY-2001-Pilates-History-Philosophy.pd $\mathrm{f}$

Madrid, B., Almeida, J. A. D., Silva, G. F., Cruz, R. D. S., Sales, M. M. D. \& Rauber, S. B. (2010) Correlação entre força de preensão manual, distância da prega palmar média e circunferência de antebraço. Revista de Educação Física, 15-19. Disponível em: http://177.38.96.106/index.php/revista/article/view/235

Maldonado, G. D. R. (2006) A educação física e o adolescente: a imagem corporal e a estética da transformação na mídia impressa. Revista Mackenzie de Educação Física e Esporte, 5(1), 59 - 76. Disponível em: http://editorarev istas.mackenzie.br/index.php/remef/article/view/1302

Miranda, V. P. N., Filgueiras, J. F., Mockdece, C. N., Teixeira, P. C. E \& Ferreira, M. E. C. (2012) Insatisfação corporal em universitários de diferentes áreas de conhecimento. Jornal Brasileiro de Psiquiatria, 61, 25-32. Disponível em: http://www.ipub.ufrj.br/portal/jbp/61/01/06\%20_JBP_61(1).pdf

Muscolino, J. E. y Cipriani, S. (2004). Pilates and the "powerhouse"-I. Journal of Bodywork and Movement Therapies, 8(1), 15-24. Disponível em: https://www.sciencedirect.com/science/article/pii/S1360859203000573

Neumark-Sztainer, D., Eisenberg, M. E., Wall, M. \& Loth, K. A. (2011) Yoga and Pilates: associations with body image and disordered-eating behaviors in a population-based sample of young adults. International Journal of Eating Disorders, 44(3), 276-80. Disponível em: https://www.ncbi.nlm.nih.gov/pmc/articles/PMC3010485/

Novaes, J. V y Vilhena, J. (2003) De cinderela a moura torta: sobre a relação mulher, beleza e feiura. Interações, 8(15), Campo Grande. Disponível em: http://www.scielo.br/scielo.php?script=sci_nlinks\&ref=000195\&pid=S1414 $-3283201200020001000017 \& \operatorname{lng}=\mathrm{pt}$

Oliveira F. P., Bosi M. L. M., Vigário P. S., \&Vieira R. S. (2003) Comportamento alimentar e imagem corporal em atletas. Revista Brasileira de Medicina do Esporte. 9(6) 348-356. Disponível em: http://www.scielo.br/pdf/\%0 D/rbme/v9n6/18934.pdf

Pilates, J. H., Miller, W. J. (1934) Your Health. Incline Village, Estados Unidos da América

Quadros, T. M. B, Gordia, A. P. M, Rebolho, C, Silva, D. A. S, Ferrari, E. P \& Petroski, E. L. (2010) Imagem corporal em universitários: associação com o estado nutricional e sexo. Motriz. 16(1).

Rossi, D. M., Morcelli, M. H., Marques, N. R., Hallal, C. Z., Gonçalves, M., ... \& Navega, M. T. (2004) Antagonist coactivation of trunk stabilizer muscles during Pilates exercises. Journal of Bodywork and Movement Therapies, 18(1). Disponível em: https://www.ncbi.nlm.nih.gov/pubmed/24411147

Sante, A.B. y Pasian, S. R. (2011) Imagem Corporal e características de personalidade de mulheres solicitantes de cirurgia plástica estética. Psicologia: Reflexão e Crítica 24 (3) 429 - 37. Disponível em: http://www.scielo.br/sc ielo.php?script $=$ sci_arttext\&pid=S0102-79722011000300003

Schilder, P. (1999) A imagem do corpo: as energias construtivas da psique. 3.ed. SãoPaulo, SP. Martins Fontes.

Serafini, G., Vargas, I. Q., Junior, L. Y. E \& Tocha, C. P. (2014) Avaliação da imagem corporal em praticantes do Método Pilates. Revista Brasileira de Reabilitação e Atividade Física 3(1).

Siler, B. (2008) Corpo Pilates, O. Grupo Editorial Summus. São Paulo, Brasil

Souza, E. F., Cantergi, D., Mendonça, A., Kennedy, C. \& Loss, J. F. (2012) Electromyographic analysis of the rectus femoris and rectus abdominis muscles during performance of the hundred and teaser pilates exercises. Revista Brasileira de Medicina do Esporte 18(2) 105-108. Disponível em: http://www.scielo.br/scielo.php?script=sci_a rttext\&pid=S1517-86922012000200008

Souza, C., Silveira, E. M. , Schmit, E. F. D. , Neto, E. S. W. , Costa, L. M. R. , Kennedy, C. \& Loss, J. F. (2017) Desenvolvimento de uma escala de imagem corporal para mulheres brasileiras. Revista Brasileira de Cineantropometria e Desempenho Humano, 19. Disponível em: http://www.scielo.br/scielo.php?script=sci_art text\&pid=S1980-00372017000500608\&lng=pt\&nrm=iso\&tlng=pt 
World Health Organization. (2005). Promoting mental health: concepts, emerging practice. Geneva: World Health Organization.

\section{BY-NC-SA}

